\title{
Mortalidad y factores pronósticos en pacientes hospitalizados por COVID-19 en la Unidad de Cuidados Intermedios de un hospital público de Lima, Perú
}

Karen Valenzuela Casquino ${ }^{1, a}$, Amadeo Espinoza Venero ${ }^{1, b}$, Jean Carlos Quispe Galvez *1,c

\section{RESUMEN}

Objetivo: Describir las características de los pacientes hospitalizados en la Unidad de Cuidados Intermedios del Hospital Uldarico Rocca Fernández y determinar los factores asociados a mortalidad.

Materiales y métodos: Estudio observacional, descriptivo y retrospectivo realizado en la Unidad de Cuidados Intermedios del Hospital Uldarico Rocca Fernández Lima-Perú, que incluye los pacientes hospitalizados por SARS-CoV-2 durante el periodo comprendido entre el 1 de julio al 30 de setiembre del 2020. Se revisó la historia clínica y registros hospitalarios para buscar características epidemiológicas, características clínicas, resultados de exámenes auxiliares al ingreso y el desenlace de la hospitalización.

Resultados: Se estudiaron 71 historias clínicas, las características epidemiológicas predominantes fueron sexo masculino $(80,28 \%)$ y edad promedio de 64,72 años $\pm 13,64$. La mayoría de los pacientes $(61,97 \%)$ presentaba al menos una comorbilidad, las más frecuentes fueron obesidad (35,21\%) e hipertensión arterial (32,39 \%). La media de estancia hospitalaria fue de 13 días ( \pm 12 días) y la tasa de mortalidad encontrada fue de 71,83\%.

Conclusiones: A partir de los resultados encontrados, esta investigación respalda lo siguiente: la población masculina presenta mayor riesgo de enfermar. Además, existen factores asociados a un peor pronóstico de mortalidad, como ser mayor de 60 años, ingresar por el servicio de emergencia con una saturación menor de 80 y una estancia hospitalaria prolongada.

Palabras clave: Pandemias; Coronavirus; COVID-19; Mortalidad; Pronóstico; Hospitalización (Fuente: DeCS BIREME).

\section{Mortality and prognostic factors among patients hospitalized for COVID-19 in the Intermediate Care Unit of a public hospital in Lima, Peru}

\section{ABSTRACT}

Objective: To describe the characteristics of patients hospitalized in the Intermediate Care Unit of the Hospital Uldarico Rocca Fernández and determine the factors associated with mortality.

Materials and methods: An observational, descriptive and retrospective study carried out in the Intermediate Care Unit of the Hospital Uldarico Rocca Fernández in Lima, Peru, which included patients hospitalized for SARS-CoV-2 from July 1 to September 30, 2020. Clinical records and hospital records were reviewed looking for epidemiological characteristics, clinical characteristics, results of additional tests on admission and the final outcome of hospitalization.

Results: Seventy-one (71) clinical records were studied. The predominant epidemiological characteristics were males $(80.28 \%)$ and mean age (64.72 years \pm 13.64$)$. Most of the patients $(61.97 \%)$ had at least one comorbidity, out of which the most common ones were obesity (35.21\%) and hypertension (32.39\%). The mean hospital stay was 13 days ( \pm 12 days) and the mortality rate was $71.83 \%$.

Conclusions: Based on the results, this research supports the following: the male population has a greater risk of becoming ill. In addition, there are factors associated with a worse mortality prognosis, such as age over 60 , admission at the emergency room with an oxygen saturation lower than $80 \%$, and prolonged hospital stay.

Keywords: Pandemics; Coronavirus; Coronavirus infections; Mortality; Prognosis; Hospitalization (Source: MeSH NLM).

\footnotetext{
1 Hospital Uldarico Rocca Fernández. Lima, Perú.

a Médico Cirujano, Especialista en Medicina Intensiva.

b Magister en Gestión y Docencia en Alimentación y Nutrición.

c Licenciado en Nutrición.

* Autor corresponsal.
} 


\section{INTRODUCCIÓN}

Entre los años 2002 y 2003, en Guangdong (China), fue detectado el SARS-CoV que produjo la infestación de 8098 personas en todo el mundo y provocó la muerte de 774 enfermos. La epidemia del SARS-CoV se controló, el último caso conocido ocurrió a mediados de 2004 y hasta la actualidad no se han registrado nuevos reportes $(1,2)$. En el año 2012, el coronavirus del síndrome respiratorio de Oriente Medio (MERS-CoV) se detectó por primera vez en Arabia Saudita y, hasta el 2019, se registraron 2494 infectados y 858 muertes personas en el mundo ${ }^{(3)}$. En diciembre del 2019, en la ciudad de Wuhan, China, un nuevo coronavirus fue identificado en un grupo de pacientes. Al inicio, este nuevo virus fue nombrado, tentativamente, como "2019 nuevo coronavirus" (2019-nCoV) ${ }^{(4)}$; luego, el Comité Internacional de Taxonomía de Virus (ICTV) lo ha denominado SARS- CoV-2 ${ }^{(5)}$.

El SARS-CoV-2 tiene características típicas de la familia de los coronavirus y se clasificó en el linaje beta coronavirus $2 \mathrm{~b}$ que es mucho más cercano, genéticamente, a los coronavirus de los murciélagos que al SARS humano ${ }^{(1,6)}$. Las primeras manifestaciones clínicas de los primeros pacientes de la ciudad de Wuhan eran fiebre, tos no productiva, disnea, mialgia y fatiga; además de disfunción orgánica y muerte en casos graves. En los exámenes auxiliares encontraron un recuento leucocitario normal o disminuido y evidencia radiográfica de neumonía ${ }^{(4)}$.

El 30 de enero de 2020, la Organización Mundial de la Salud (OMS) declaró a la infección por SARS-CoV-2 una emergencia internacional de salud pública ${ }^{(7)}$; y el 11 de marzo de 2020, como una pandemia ${ }^{(8)}$. En el mundo, más de un millón de personas está infectado y los fallecidos son más de 50 000. Los países más afectados han sido Italia, España y, en los últimos días, Estados Unidos de América; en Sudamérica, Brasil y Ecuador son las naciones con mayor número de muertes ${ }^{(9)}$. En el Perú, al inicio de este estudio, se habían reportado 285213 casos y 9677 fallecidos ${ }^{(10)}$.

En nuestro país, el primer caso de COVID-19 fue identificado el 6 de marzo de 2020. A partir de este momento, comenzó la transmisión comunitaria (11). La enfermedad se propagó de manera exponencial, lo cual llevó al Gobierno Peruano a declarar la emergencia sanitaria ${ }^{(12)}$ y establecer hospitales dedicados exclusivamente a tratar pacientes con COVID-19; además, se implementaron las teleconsultas ${ }^{(13)}$.

El Hospital Uldarico Rocca Fernández, ubicado en el distrito de Villa El Salvador, es un establecimiento de salud perteneciente a la Red Prestacional Rebagliati que brinda atención a los afiliados al Seguro Social de Salud (EsSalud) del Perú.

En este estudio determinaremos los factores asociados a la mortalidad y los desenlaces de los pacientes hospitalizados por COVID-19 en la Unidad de Cuidados Intermedios del Hospital Uldarico Rocca Fernández.

\section{MATERIALES Y MÉTODOS}

\section{Diseño y población}

Estudio observacional de cohorte y retrospectivo, a partir de la revisión de las historias clínicas de los pacientes hospitalizados por COVID-19 en la Unidad de Cuidados Intermedios del Hospital Uldarico Rocca Fernández (HURF) que pertenece a la Red Desconcentrada III Rebagliati y está ubicado en el distrito de Villa El Salvador en Lima, Perú.

En la investigación fueron incluidos los pacientes hospitalizados en la UCIN diagnosticados con COVID-19 entre el 1 de julio al 30 setiembre del 2020. Se excluyeron a los pacientes con desenlace incierto (transferencias a otros hospitales o retiros voluntarios).

\section{Variables}

Los antecedentes patológicos que se consideraron en este estudio fueron la hipertensión arterial y la diabetes mellitus (en ambos casos, pacientes con diagnóstico previo al ingreso por el Servicio de Emergencia o que recibían o precisaron tratamiento farmacológico antihipertensivo o antidiabético). La anemia al ingreso se definió como la concentración de hemoglobina por debajo del nivel normal establecido de acuerdo a la escala de la OMS: $\geq 12 \mathrm{~g} / \mathrm{dL}$ para las mujeres, $y \geq 13 \mathrm{~g} / \mathrm{dL}$ para los hombres.

La estancia hospitalaria se definió como corta, si fue menor de 5 días (inferior al percentil 25), intermedia, de 5 a 17 días (percentil 25 al 75), y prolongada si fue mayor de 17 días (superior al percentil 75). Los grupos etarios fueron calificados en joven (18 a 29 años), adulto (30 a 59) y adulto mayor (mayor de 60 años).

\section{Análisis estadístico}

La información fue recolectada en un formulario electrónico creado en Microsoft Excel, que incluyó características demográficas, características clínicas, resultados de exámenes auxiliares al ingreso y el desenlace de la hospitalización. Posteriormente, los datos se importaron a Past Versión 2.17 para realizar el análisis estadístico.

Las variables categóricas se reportaron con frecuencias y porcentajes, y las variables continuas con medianas, con su respectivo rango intercuartil (RIC). La hipótesis de normalidad fue evaluada con los test de Shapiro-Wilk. Para la comparación de variables empleamos la prueba de $X^{2}$ para las variables cualitativas, y la $t$ de Student para las cuantitativas. Además, los principales factores independientes pronósticos de mortalidad hospitalaria fueron identificados mediante la regresión logística 
múltiple. Un valor de $p$ menor de 0,05 se consideró estadísticamente significativo.

\section{Consideraciones éticas}

El protocolo del estudio fue autorizado por la Dirección General del HURF. La identidad de los participantes no fue registrada. Esta información es de acceso público y abierto, por lo que no se requiere de una aprobación ética.

\section{RESULTADOS}

Durante el periodo de estudio, se identificaron 74 historias clínicas pertenecientes a pacientes adultos hospitalizados por COVID-19 en la Unidad de Cuidados Intermedios del Hospital Uldarico Rocca Fernández. De ellos, 71 cumplieron con los criterios de inclusión, de los cuales 51 fallecieron, lo que mostró una tasa de mortalidad de $71,83 \%$. Tres pacientes fueron excluidos por no presentar información sobre el desenlace de la enfermedad (Figura 1).

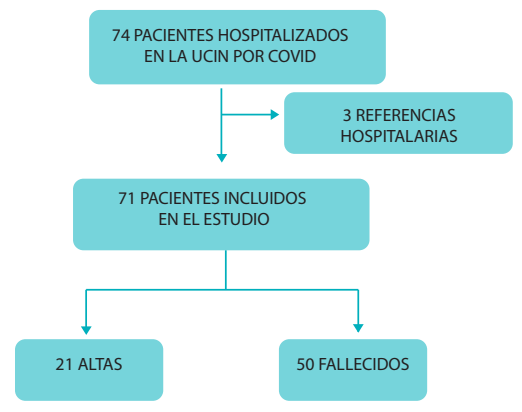

Figura 1. Registro de pacientes y desenlace de la hospitalización. Hospital Uldarico Rocca Fernández

La edad media de los pacientes fue de 64,72 $\pm 13,64$ años. Cincuenta y siete pacientes fueron hombres (80,28 \%) (Figura 2 ).

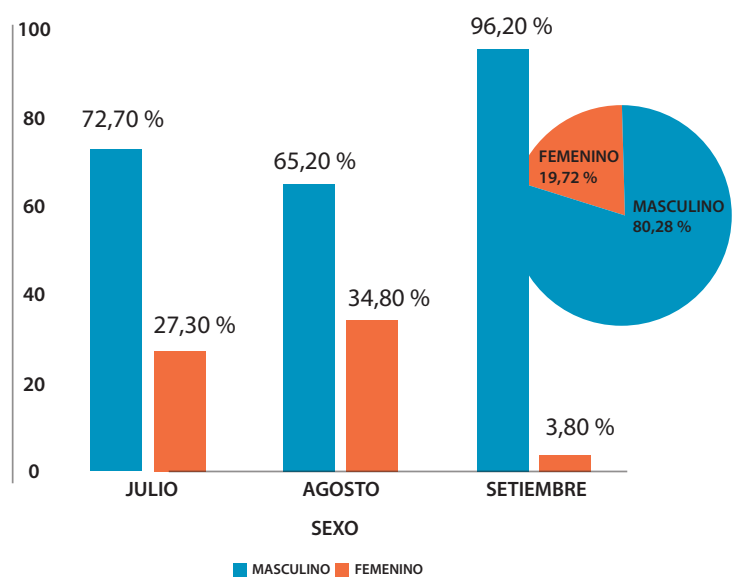

Figura 2. Distribución de pacientes hospitalizados por COVID-19 por mes de ingreso y sexo. Hospital Uldarico Rocca Fernández

Respecto al mayor porcentaje de hospitalizados según grupo etario, los adultos mayores fueron el grupo más numeroso $(64,79 \%)$, seguido de los adultos $(33,80 \%)$ y los jóvenes $(1,41 \%)$. La mayoría de los pacientes $(61,97 \%)$ presentaba al menos una comorbilidad, principalmente obesidad $(35,21 \%)$ y, en segundo lugar, hipertensión arterial (32,39\%). Adicionalmente, un 36,23\% de los participantes acudían con $\mathrm{SatO}_{2}$ menor a $80 \%$. En los exámenes auxiliares encontramos anemia en un $15,49 \%$. La mediana de estancia hospitalaria reportada era de 13 días ( \pm 12 días) hasta el desenlace (Tabla 1 ). 
Tabla 1. Características clínicas y demográficas al ingreso de los pacientes con COVID-19. Hospital Uldarico Rocca Fernández

\begin{tabular}{|lccc|}
\hline \multicolumn{1}{|c}{ Variable } & Hombres & Mujeres & Total n (\%) \\
\hline Edad (años) & $67( \pm 12,2)$ & $55,6( \pm 15,7)$ & $64,7( \pm 13,64)$ \\
\hline Hemoglobina (g/dl) & $13,9( \pm 1,6)$ & $13,5( \pm 1,8)$ & $13,8( \pm 1,5)$ \\
\hline Estancia hospitalaria (días) & $12,5( \pm 12,7)$ & $15,5( \pm 11,3)$ & $13,1( \pm 12,4)$ \\
\hline Saturación de oxígeno al ingreso (\%) & $80,2( \pm 10,1)$ & $77,8( \pm 16,5)$ & $79,7( \pm 11,5)$ \\
\hline Comorbilidades & & & \\
\hline Antecedente obesidad & $15(60 \%)$ & $10(40 \%)$ & $25(35,21 \%)$ \\
\hline Antecedente hipertensión arterial & $20(87 \%)$ & $3(13 \%)$ & $23(32,39 \%)$ \\
\hline Antecedente diabetes mellitus & $13(76,5 \%)$ & $4(23,5 \%)$ & $17(23,94 \%)$ \\
\hline Número de comorbilidades & & & \\
\hline Ninguna & $25(93 \%)$ & $2(7 \%)$ & $27(38,03 \%)$ \\
\hline Una & $18(69 \%)$ & $8(31 \%)$ & $26(36,62 \%)$ \\
\hline Dos & $12(80 \%)$ & $3(20 \%)$ & $15(21,13 \%)$ \\
\hline Tres & $2(66,67 \%)$ & $1(33,33 \%)$ & $3(4,23 \%)$ \\
\hline
\end{tabular}

La tasa de mortalidad encontrada en este estudio fue de $71,83 \%$. El 76,47 \% de los fallecidos fueron adultos mayores y el versus, $23,53 \%$, eran adultos. Es resaltante la asociación entre mortalidad y la edad mayor de 60 años $(p<0,05)$.

Otro análisis mostró que el $82,35 \%$ de los fallecidos eran hombres $(n=42)$ y $17,65 \%$, mujeres $(n=9)$. Esta diferencia no fue estadísticamente significativa $(p>0,05)$.
El valor de la saturación de oxígeno $<80 \%$ tomada al ingreso se asoció con una mayor mortalidad, en comparación al grupo de los sobrevivientes $(p<0,0025)$. El mayor porcentaje de pacientes hospitalizados que fallecieron $(49,02 \%, n=25)$ tuvo una estancia hospitalaria intermedia $(p<0,025)$.

De los fallecidos, 19 (37,25\%) tenían el antecedente de hipertensión arterial $(p>0,1), 15(29,41 \%)$ padecían de diabetes mellitus $(p>0,05)$ y el $33,33 \%$ eran obesos $(p>0,5)$ (Tabla 2).

Tabla 2. Factores pronósticos de mortalidad hospitalaria en pacientes con COVID-19. Hospital Uldarico Rocca Fernández

\begin{tabular}{|c|c|c|c|c|}
\hline Factor pronóstico & Fallecidos & Altas & Total & $p$ \\
\hline \multicolumn{5}{|l|}{ Grupo etario } \\
\hline Joven & 0 & 1 & 1 & $<0,0025$ \\
\hline Adulto & 12 & 12 & 24 & $<0,0025$ \\
\hline Adulto mayor & 39 & 7 & 46 & $<0,0025$ \\
\hline \multicolumn{5}{|l|}{ Sexo } \\
\hline Masculino & 42 & 15 & 57 & $>0,1$ \\
\hline Femenino & 9 & 5 & 14 & $>0,1$ \\
\hline \multicolumn{5}{|l|}{ Saturación de oxígeno } \\
\hline \multicolumn{5}{|l|}{ al ingreso } \\
\hline$>90$ & 1 & 5 & 6 & $<0,0025$ \\
\hline $85-90$ & 14 & 9 & 23 & $<0,0025$ \\
\hline $80-84$ & 14 & 1 & 15 & $<0,0025$ \\
\hline$<80$ & 20 & 5 & 25 & $<0,0025$ \\
\hline
\end{tabular}


Mortalidad y factores pronósticos en pacientes hospitalizados por COVID-19 en la

Unidad de Cuidados Intermedios de un hospital público de Lima, Perú

\begin{tabular}{|c|c|c|c|c|}
\hline Factor pronóstico & Fallecidos & Altas & Total & $p$ \\
\hline \multicolumn{5}{|l|}{ Estancia hospitalaria } \\
\hline Corta & 16 & 0 & 16 & $<0,025$ \\
\hline Intermedia & 25 & 12 & 37 & $<0,025$ \\
\hline Prolongada & 10 & 8 & 18 & $<0,025$ \\
\hline \multicolumn{5}{|l|}{ Antecedente de HTA } \\
\hline Con HTA & 19 & 4 & 23 & $>0,1$ \\
\hline Sin HTA & 32 & 16 & 48 & $>0,1$ \\
\hline \multicolumn{5}{|l|}{ Antecedente de DM } \\
\hline Con DM & 15 & 2 & 17 & $>0,05$ \\
\hline Sin DM & 36 & 18 & 54 & $>0,05$ \\
\hline \multicolumn{5}{|c|}{ Diagnóstico de obesidad } \\
\hline Obesos & 17 & 8 & 25 & $>0,5$ \\
\hline No obesos & 34 & 12 & 46 & $>0,5$ \\
\hline \multicolumn{5}{|c|}{ Número de comorbilidades } \\
\hline Ninguna & 19 & 8 & 27 & $>0,2$ \\
\hline Una & 16 & 10 & 26 & $>0,2$ \\
\hline Dos & 13 & 2 & 15 & $>0,2$ \\
\hline Tres & 3 & 0 & 3 & $>0,2$ \\
\hline
\end{tabular}

\section{DISCUSIÓN}

En el presente estudio, la edad promedio de los pacientes con COVID-19 ingresados a la Unidad de Cuidados Intermedios fue de $64,72 \pm 13,64$ años, valor que es cercano al que Mejía et al. (17) encontraron en sus pacientes hospitalizados por COVID-19 (59 años).

Nuestro estudio muestra que el $64,79 \%$ de los casos correspondían a adultos mayores de 60 años, lo cual es similar a lo reportado en China, EE. UU. y en estudios peruanos ${ }^{(14-17)}$. Las comorbilidades más frecuentes fueron la obesidad $(35,21 \%)$, la hipertensión arterial $(32,39 \%)$ y diabetes mellitus $(23,94 \%)$, resultados similares a los obtenidos en un hospital de referencia nacional de Lima(16); estas comorbilidades han sido mencionadas como factores de riesgo para desarrollar la forma grave de enfermedad ${ }^{(18,19)}$.

La mayoría de pacientes hospitalizados $(80,28 \%)$ fueron hombres $(p<0,05)$, lo cual sugiere un mayor riesgo de enfermar en la población masculina. Esta información concuerda con los hallazgos de Acosta et al. (15), quienes encontraron una mayor frecuencia de ingresos en pacientes del sexo masculino (76 \%). Sin embargo, al analizar la relación entre el sexo y la mortalidad no se encontró significancia estadística $(p>0,1)$.

La tasa de mortalidad encontrada en esta investigación fue de $71,83 \%$, este valor es mayor al $49,6 \%$ que se reportó en los pacientes hospitalizados por COVID-19 de otro hospital de Lima ${ }^{(17)}$ y muy superior a la tasa de mortalidad hallada en estudios realizados en Wuhan, China $(30 \%)^{(4,20)}$.

Cabe destacar que, en este estudio, ninguna de las tres morbilidades más frecuentes (obesidad, hipertensión arterial y diabetes) tuvo relación significativa con la mortalidad ( $p>0,5, \quad p>0,1, \quad p>0,05$, respectivamente). Asimismo, un estudio realizado en España (14) mostró que las personas mayores de 65 años presentaron una fuerte correlación con la mortalidad $(p<0,001)$. En nuestra investigación, un elevado porcentaje de los fallecidos $(76,47 \%)$ fueron adultos mayores $(p<0,0025)$.

El estudio realizado por Mejía et al. (17) señaló que los pacientes son hospitalizados de forma tardía, cuando la hipoxemia ya es significativa. En ese sentido, nuestros hallazgos también sugieren que la saturación al ingreso y el tiempo de la estancia hospitalaria podrían ser factores pronósticos de mortalidad $(p<0,025$ y $p<0,0025)$.

En conclusión, este estudio respalda la idea de que la población masculina presenta un mayor riesgo de enfermar. Además, existen factores asociados a un peor pronóstico de mortalidad, como ser mayor de 60 años, llegar a la emergencia con una saturación de oxígeno menor de $80,00 \%$ al ingreso y una estancia hospitalaria prolongada. 
Contribuciones de los autores: Jean Carlos Quispe Galvez: Concepción, recolección de datos, análisis e interpretación de datos y revisión del artículo. Amadeo Ivanovich Espinoza Venero: Concepción y el diseño del artículo, análisis e interpretación de datos, revisión crítica del artículo y aprobación de la versión final. Karen Valenzuela Casquino: Análisis e interpretación de datos, revisión crítica del artículo y aprobación de la versión final.

Fuentes de financiamiento: El artículo ha sido financiado por los autores.

Conflictos de interés: Los autores declaran no tener conflictos de interés.

\section{REFERENCIAS BIBLIOGRÁFICAS}

1. Velavan T, Meyer C. The Covid-19 epidemic. Trop Med Int Health. 2020; 25(3): 278-80.

2. Hui D, Azhar E, Madani T, Ntoumi F, Kock R, Dar O. The continuing 2019-nCoV epidemic threat of novel coronaviruses to global health. The latest 2019 novel coronavirus outbreak in Wuhan, China. Int J Infect Dis. 2020; 91: 264-6.

3. Zaki A, Van Boheemen S, Bestebroer T, Osterhaus A, Fouchier R. Isolation of a novel coronavirus from a man with pneumonia in Saudi Arabia. N Engl J Med. 2012; 367: 1814-20.

4. Huang C, Wang Y, Li X, Ren L, Zhao J, Hu Y, et al. Clinical features of patients infected with 2019 novel coronavirus in Wuhan, China. Lancet. 2020; 395(10223): 497-506.

5. Firas R, Mazhar A, Ghena K, Dunia S, Amjad A. SARS-CoV2 and Coronavirus Disease 2019: What we know so far. Pathogens. 2020; 9(3): 231.

6. Lu R, Zhao X, Li J, Niu P, Yang B, Wu H. Genomic characterisation and epidemiology of 2019 novel coronavirus: implications for virus origins and receptor binding. Lancet. 2020; 395(10224): 565-74.

7. Organización Mundial de la Salud (OMS). Declaración sobre la segunda reunión del Comité de Emergencias del Reglamento Sanitario Internacional (2005) acerca del brote del nuevo coronavirus (2019nCoV). [Internet]. 2020. Disponible en: https://www.who.int/es/ news/item/30-01-2020-statement-on-the-second-meeting-of-theinternational-health-regulations-(2005)-emergency-committeeregarding-the-outbreak-of-novel-coronavirus-(2019-ncov)

8. Organización Mundial de Salud (OMS). Alocución de apertura del Director General de la OMS en la rueda de prensa sobre la COVID-19 celebrada el 11 de marzo de 2020. [Internet]. 2020. Disponible en: https://www.who.int/es/dg/speeches/detail/who-directorgeneral-s-opening-remarks-at-the-media-briefing-on-covid-19---11march-2020

9. World Health Organization. Coronavirus disease 2019 (COVID-19) Situation Report - 77 [Internet]. 2020. Disponible en: https://apps. who.int/iris/handle/10665/331718?locale-attribute=es\&

10. Ministerio de Salud (MINSA). Minsa: Casos confirmados por Coronavirus Covid-19 ascienden a 285213 en el Perú (Comunicado $\mathrm{N}^{\circ}$ 153). [Internet]. Perú; 2020. Disponible en: https://www.gob. $\mathrm{pe} /$ institucion/minsa/noticias/189369-minsa-casos-confirmadospor-coronavirus-covid-19-ascienden-a-285-213-en-el-perucomunicado-n-153

11. Ministerio de Salud (MINSA). Alerta epidemiológica $N^{\circ} 13$ - Coronavirus (COVID-19). [Internet]. Perú; 2020. Disponible en: https://www. gob.pe/institucion/minsa/informes-publicaciones/462473-alertaepidemiologica-n-13-coronavirus-covid-19
12. Presidencia de la República. Decreto Supremo que declara en Emergencia Sanitaria a nivel nacional por el plazo de noventa (90) días calendario y dicta medidas de prevención y control del COVID-19 [Internet]. Diario Oficial del Bicentenario "El Peruano". 2020. Disponible en: https://busquedas.elperuano.pe/normaslegales/ decreto-supremo-que-declara-en-emergencia-sanitaria-aniveldecreto-supremo-n-008-2020-sa-1863981-2/

13. Directiva Administrativa para la Implementación y Desarrollo de los Servicios de Teleorientación y Telemonitoreo. Resolución Ministerial N¹46-2020-MINSA 2020 (31-03-20) [Internet]. Disponible en: https: / / www.gob.pe/institucion/minsa/normas-legales/466078. 146-2020-minsa.

14. Medeiros Figueiredo A, Daponte-Codina A, Moreira Marculino DC, Toledo Vianna RP, Costa de Lima K, Gil-García E. Factores asociados a la incidencia y la mortalidad por COVID-19 en las comunidades autónomas. Gac Sanit. 2020.

15. Acosta G, Escobar G, Bernaola G, Alfaro J, Taype W, Marcos C, et al. Caracterización de pacientes con COVID-19 grave atendidos en un hospital de referencia nacional del Perú. Rev Peru Med Exp Salud Publica. 2020; 37(2): 253-8.

16. Llaro S, Gamarra V, Campos C. Características clínico-epidemiológicas y análisis de sobrevida en fallecidos por COVID-19 atendidos en establecimientos de la Red Sabogal-Callao 2020. Horiz Med. 2020; 20(2): e1229.

17. Mejía F, Medina C, Cornejo E, Morello E, Vásquez S, Alave J, et al. Características clínicas y factores asociados a mortalidad en pacientes adultos hospitalizados por COVID-19 en un hospital público de Lima, Perú. Public Health. 2020.

18. Zhang J-J, Dong X, Cao Y-Y, Yuan Y-D, Yang Y-B, Yan T-Q, et al. Clinical characteristics of 140 patients infected with SARSCoV-2 in Wuhan, China. Allergy. 2020; 75(7): 1730-41.

19. Yang J, Zheng Y, Gou X, Pu K, Chen Z, Guo Q, et al. Prevalence of comorbidities and its effects in patients infected with SARS-CoV-2: a systematic review and meta-analysis. Int J Infect Dis. 2020; 94: 91-5.

20. Yang X, Yu Y, Xu J, Shu H, Xia J, Liu H, et al. Clinical course and outcomes of critically ill patients with SARS-CoV-2 pneumonia in Wuhan, China: a single-centered, retrospective, observational study. Lancet. 2020; 8(5): 475-81.

\section{Correspondencia:}

Jean Carlos Quispe Galvez

Dirección: Av. Las Palmas Mz T Lote 27 - Pachacamac. Lima, Perú.

Teléfono: 937433122

Correo electrónico: quispegalvezjk@gmail.com

Recibido: 07 de diciembre de 2020 Evaluado: 30 de diciembre de 2020 Aprobado: 11 de enero de 2020.

(c) La revista. Publicado por Universidad de San Martín de Porres, Perú (cc) Br Licencia de Creative Commons Artículo en acceso abierto bajo términos de Licencia Creative Commons Atribución 4.0 Internacional. (http://creativecommons.org/licenses/by/4.0/)

\section{ORCID iDs}

Karen Valenzuela Casquino https://orcid.org/0000-0002-0730-0693 Amadeo Ivanovich Espinoza V. C https: / / orcid.org/0000-0001-8612-4351 Jean Carlos Quispe Galvez $\quad$ https://orcid.org/0000-0003-1769-2288 